\title{
The effect of emicizumab prophylaxis on health-related
outcomes in persons with haemophilia A with inhibitors:
HAVEN 1 Study \\ The effect of emicizumab prophylaxis on health-related
outcomes in persons with haemophilia A with inhibitors:
HAVEN 1 Study HAVEN 1 Study
}

\author{
Johannes Oldenburg ${ }^{1}$ (D) | Johnny N. Mahlangu² (D) | Willem Bujan ${ }^{3}$ | Peter Trask ${ }^{4}$ | \\ Michael U. Callaghan' ${ }^{5}$ | Guy Young6 ${ }^{6}$ | Elina Asikanius ${ }^{7}$ | Flora Peyvandi ${ }^{8}$ (D) | \\ Elena Santagostino $^{8}$ | Rebecca Kruse-Jarres ${ }^{9}$ (D) | Claude Negrier ${ }^{10}$ | Craig Kessler ${ }^{11}$ | \\ Jin $\mathrm{Xu}^{4}$ | Jerzy Windyga ${ }^{12}$ (i) | Midori Shima ${ }^{13}$ | Sylvia von Mackensen ${ }^{14}$ (D) \\ ${ }^{1}$ Department of Experimental Haematology and Transfusion Medicine, Universitätsklinikum University Clinic Bonn, Bonn, Germany \\ ${ }^{2}$ Haemophilia Comprehensive Care Centre, Faculty of Health Sciences, University of the Witwatersrand and NHLS, Johannesburg, South Africa \\ ${ }^{3}$ Instituto de Costarricense de Investigaciones Científicas, San Jose, Costa Rica \\ ${ }^{4}$ Genentech, Inc., South San Francisco, California \\ ${ }^{5}$ Children's Hospital of Michigan, Detroit Medical Center, Detroit, Michigan \\ ${ }^{6}$ Children's Hospital Los Angeles, University of Southern California Keck School of Medicine, Los Angeles, California \\ ${ }^{7}$ F. Hoffmann-La Roche Ltd, Basel, Switzerland \\ ${ }^{8}$ IRCCS Ca' Granda Foundation, Ospedale Maggiore Policlinico, Milan, Italy \\ ${ }^{9}$ Washington Center for Bleeding Disorders at BloodWorks NW, University of Washington, Seattle, Washington \\ ${ }^{10}$ Louis Pradel University Hospital, Lyon, France \\ ${ }^{11}$ Georgetown University Medical Center, N.W., Washington, District of Columbia \\ ${ }^{12}$ Department of Disorders of Hemostasis and Internal Medicine, Institute of Hematology and Transfusion Medicine, Warsaw, Poland \\ ${ }^{13}$ Department of Pediatrics, Nara Medical University, Kashihara, Japan \\ ${ }^{14}$ University Medical Centre Hamburg-Eppendorf, Hamburg, Germany
}

\section{Correspondence}

Sylvia von Mackensen, Department of Medical Psychology, University Medical Centre Hamburg-Eppendorf, Hamburg, Germany.

Email: s.mackensen@uke.uni-hamburg.de

Funding information

This study was funded by F. Hoffmann-La Roche Ltd.
Introduction: Persons with haemophilia A (PwHA) with inhibitors to factor VIII often experience decreased health-related outcomes. In HAVEN 1 (NCT02622321), there was a statistically significant reduction in bleeding with emicizumab prophylaxis versus no prophylaxis.

Aim: Describe health-related outcomes in PwHA with inhibitors in HAVEN 1. Methods: PwHA with inhibitors aged $\geq 12$ years previously on episodic bypassing agents (BPAs) were randomized to emicizumab prophylaxis (Arm $A ; n=35$ ) or no prophylaxis (Arm $B ; n=18$ ); participants previously on BPA prophylaxis received emicizumab prophylaxis (Arm C; $n=49$ ). Health-related outcomes assessed at baseline and monthly thereafter: Haemophilia Quality of Life Questionnaire for Adults (Haem-A-QoL), Haemophilia-specific Quality of Life Questionnaire for Children Short Form (Haemo-QoL SF), EuroQol 5-Dimensions 5-Levels (EQ-5D-5L) index utility score (IUS) and visual analogue scale (EQ-VAS) and work/school days. Days hospitalized also recorded. 
Results: At week 25, differences (ANCOVA) in adjusted mean scores ( $95 \%$ confidence interval) favoured Arm A versus B for Haem-A-QoL "Total" score (14.0 [5.6, 22.5]; $P=0.002)$ and "Physical Health" (21.6 [7.9, 35.2]; $P=0.003)$; EQ-VAS (-9.7 [-17.6, -1.82]; $P=0.017)$; and IUS (-0.16 [-0.25, -0.07]; $P=0.001)$; mean scores are comparable in Arms $A$ and $C$. Throughout the study, a greater proportion of participants on emicizumab prophylaxis than no prophylaxis exceeded questionnaire-specific responder thresholds. Mean proportion of missed work days and number of days hospitalized were lower with emicizumab prophylaxis than no prophylaxis.

Conclusions: In PwHA with inhibitors, emicizumab prophylaxis was associated with substantial and meaningful improvements in health-related outcomes.

KEYWORDS

emicizumab, factor VIII inhibitors, haemophilia A, health-related quality of life, prophylaxis

\section{1 | INTRODUCTION}

Despite advances in the treatment of haemophilia $A$ in the past decades, ${ }^{1}$ current standard approaches continue to require frequent and time-consuming intravenous infusions of factor VIII (FVIII) concentrates. $^{1-3}$ Up to $20 \%$ of persons with haemophilia A (PwHA) develop alloantibodies (inhibitors) to FVIII treatments, typically within the first 10-15 days of treatment. ${ }^{4,5}$ Inhibitors are classified as low titre $(<5$ Bethesda units $[\mathrm{BU}] / \mathrm{mL}$ ) or high titre $(>5 \mathrm{BU} / \mathrm{mL}$ at least once) based on the highest documented inhibitor level and the occurrence of an anamnestic response after re-exposure to factor concentrate. ${ }^{6}$ Among PwHA with inhibitors, therapeutic options have been more limited, particularly burdensome and suboptimally effective. ${ }^{7-12}$ Current guidelines recommend immune tolerance induction (ITI) to eliminate high-titre inhibitors as first-line treatment in these individuals, which can enable effective replacement therapy and make prophylaxis feasible in order to prevent or lessen the negative impact of persistent inhibitors on patients' morbidity and quality of life. ${ }^{13-18}$ However, ITI is associated with high costs, involves frequent, prolonged infusions and is not always effective. ${ }^{19,20}$ Bleeding in PwHA with inhibitors requires bypassing agents (BPAs; ie, activated prothrombin complex concentrates, recombinant factor VIla). ${ }^{12}$

Until recently, the only prophylaxis regimens for $\mathrm{PwHA}$ with inhibitors with acceptable outcomes required intravenous infusions of BPAs every other day, with dosing regimens varying from three times a week to daily in clinical practice. ${ }^{10-12,21}$ Despite BPA prophylaxis, PwHA with inhibitors have significant breakthrough bleeding, ${ }^{10,12}$ and often experience poor health-related quality of life (HRQoL), increased health care costs, joint pain and other orthopaedic issues, and missed days of work and school due to bleeding. ${ }^{22-26}$

Emicizumab (HEMLIBRA ${ }^{\circledR}$; F. Hoffmann-La Roche, Basel, Switzerland) was developed to address the unmet needs of PwHA with inhibitors for effective, less burdensome prophylaxis. Emicizumab is a humanized bispecific antibody that bridges activated factor $\mathrm{IX}$ and factor $\mathrm{X}$, restoring the function of missing activated FVIII and haemostasis even in the presence of FVIII inhibitors.
High subcutaneous bioavailability ${ }^{27}$ and a 4- to 5 -week half-life ${ }^{28}$ allow emicizumab to be administered subcutaneously once weekly. ${ }^{29}$ Emicizumab has been approved in several countries for prophylaxis to prevent or reduce bleeding frequency in adult and paediatric PwHA with FVIII inhibitors.

In the HAVEN 1 Phase 3 clinical study of PwHA with inhibitors (NCT02622321), emicizumab prophylaxis was associated with statistically significant and clinically meaningful reductions in bleeding versus no prophylaxis. Annualized bleeding rate ( $95 \%$ confidence interval $[\mathrm{Cl}])$ for treated bleeds was $2.9(1.7,5.0)$ with emicizumab prophylaxis and $23.3(12.3,43.9)$ with no prophylaxis (episodic BPAs only); representing an $87 \%$ difference in favour of emicizumab prophylaxis (risk ratio: $0.13 ; P<0.001$ ). ${ }^{29}$ Zero-treated bleeding events were experienced by $63 \%$ on emicizumab prophylaxis and $6 \%$ on no prophylaxis. In an intra-individual comparison among those previously using BPA prophylaxis in a prospective non-interventional study (NIS; NCT02476942), emicizumab prophylaxis resulted in a $79 \%$ decrease in bleeding rates $(P<0.001) .{ }^{29}$

The purpose of the present paper is to provide a detailed description of HRQoL and health status outcomes in PwHA with inhibitors in HAVEN 1.

\section{2 | MATERIALS AND METHODS}

HAVEN 1 was a multicentre, open-label, randomized, Phase 3 trial conducted in 43 centres in 14 countries worldwide. The protocol was approved by the institutional review board at each centre and adhered to Good Clinical Practice guidelines. The protocol for the HAVEN 1 clinical trial has been described previously ${ }^{29}$ and is summarized briefly here and in Figure 1.

PwHA (any severity) with inhibitors aged $\geq 12$ years were enrolled if they had a history of high-titre FVIII inhibitors ( $\geq 5 \mathrm{BU} / \mathrm{mL}$ ), had received BPAs to treat or control bleeding for $\geq 24$ weeks before study entry and, in the previous 24 weeks, had $\geq 6$ bleeds while on episodic BPAs or $\geq 2$ bleeds while on prophylactic BPAs. 
FIGURE 1 HAVEN 1 Study design. BPA, bypassing agent; PwHA, persons with haemophilia A; R, randomization. ${ }^{a}$ Non-interventional study (NCT02476942) collected real-world data on bleed rates and standard-of-care treatments in $\mathrm{PwHA}$ with inhibitors; participants subsequently had the option to enrol into HAVEN 1. ${ }^{b}$ Participants unable to enrol into Arms A, $\mathrm{B}$ or $\mathrm{C}$ before they closed to enrolment were enrolled in Arm D for compassionate reasons

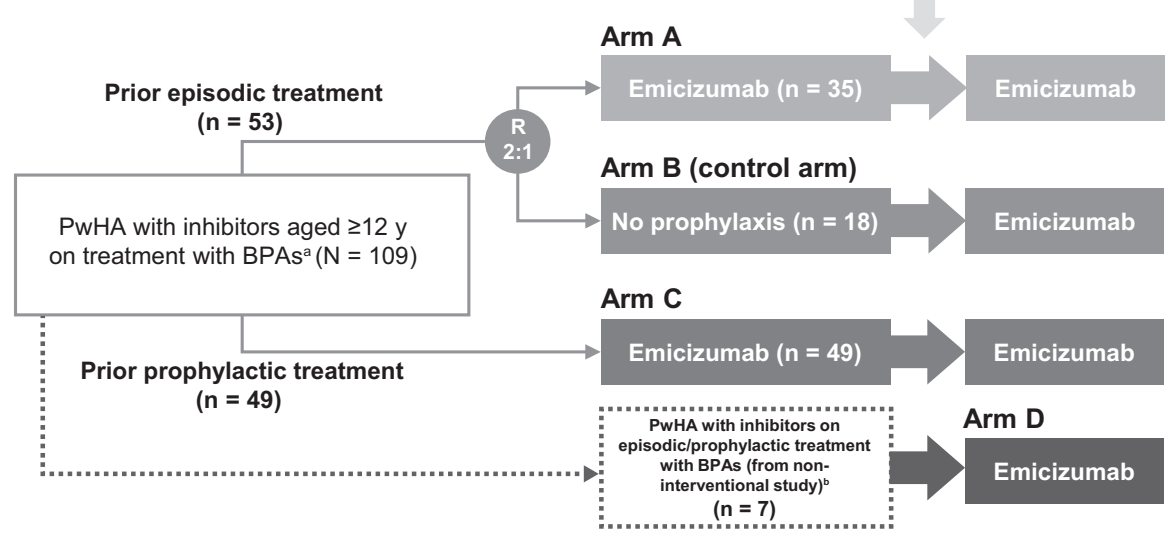

"Family," "Friends," "Other People," "Sports \& School," "Dealing with Haemophilia" and "Treatment"), each scored separately and combined to create a "Total" score. Response options ranged from "Never" (1) to "Always" (5) on a 5-point Likert scale. Some items of the domains "View of Yourself", "Friends," "Sports \& School" and "Dealing with Haemophilia" were reverse-scored as above.

For both Haem-A-QoL and Haemo-QoL SF scales, participants were asked to consider their experience in the previous 4 weeks when responding. On both scales, all domain scores were transformed to a 0-100 scale with higher scores indicating greater impairment.

\subsection{2 | Health status}

Health status was assessed using the European Quality of Life 5Dimensions 5-levels (EQ-5D-5L) questionnaire and visual analogue scale (EQ-VAS). ${ }^{34,35}$ The five dimensions of the EQ-5D-5L assess mobility, self-care, usual activities, pain/discomfort and anxiety/depression; each with five levels of severity ranging from "no problems" to "extreme problems". ${ }^{35,36}$ The five dimensions were combined into an index utility score (IUS) using the UK crosswalk value set; scores range from -0.594 (extreme problems on all dimensions) to 1 (no problems on all dimensions). ${ }^{37}$ On the EQ-VAS, participants indicated their health status on a thermometer ranging from 0 (worst imaginable) to 100 (best imaginable). For both EQ-5D-5L and EQVAS, participants were asked to report the severity experienced on the day the questionnaire was completed.

\subsubsection{Work and school absences}

Every 4 weeks, participants were asked to report how many days of work or school they missed during the previous 4 weeks due to haemophilia A, and how many days they should have been at work or school.

\subsection{4 | Hospitalizations}

Hospitalizations were recorded in the serious adverse events page of the electronic case report forms (eCRFs) by investigators. 8-17 years). ${ }^{33}$ The 35 -item measure for age group II is composed of nine domains ("Physical Health," "Feelings," "View of Yourself," 
TAB LE 1 Participant demographics and baseline characteristics ${ }^{29}$

\begin{tabular}{|c|c|c|c|}
\hline \multirow[b]{2}{*}{ Characteristic } & \multicolumn{2}{|c|}{ Previously on episodic BPAs ${ }^{a}$} & \multirow{2}{*}{$\begin{array}{l}\begin{array}{l}\text { Previously on } \\
\text { prophylactic BPAs }\end{array} \\
\text { Arm C: Emicizumab } \\
\text { prophylaxis }(n=49)\end{array}$} \\
\hline & $\begin{array}{l}\text { Arm A: Emicizumab } \\
\text { prophylaxis }(n=35)\end{array}$ & Arm B: No prophylaxis $(n=18)$ & \\
\hline Median age, y (range) & $38.0(12-68)$ & $35.5(13-65)$ & $17.0(12-75)$ \\
\hline \multicolumn{4}{|l|}{ Age groups } \\
\hline$\geq 18$ y, n (\%) & $31(88.6)$ & $16(88.9)$ & $23(46.9)$ \\
\hline \multicolumn{4}{|l|}{ Race, n (\%) } \\
\hline Asian & $10(28.6)$ & $3(16.7)$ & $8(1.36)$ \\
\hline Black or African American & $4(11.4)$ & $4(22.2)$ & $3(6.1)$ \\
\hline White & $21(60.0)$ & $10(55.6)$ & $33(67.3)$ \\
\hline$\geq 9$ & $24(68.6)$ & $13(72.2)$ & $26(53.1)$ \\
\hline Severe haemophilia at baseline, $\mathrm{n}(\%)$ & $31(88.6)$ & $18(100.0)$ & $47(95.9)$ \\
\hline Previously treated with ITI, n (\%) & $14(40.0)$ & 7 (38.9) & $33(67.3)$ \\
\hline $\begin{array}{l}\text { Highest historical inhibitor titre levels, }{ }^{\mathrm{C}} \text { median } \\
\text { BU (range) }\end{array}$ & $288.9(5-1570)$ & $706.8(18-4500)$ & $815.7(11-5000)$ \\
\hline
\end{tabular}

BPA, bypassing agents, bypassing agent; BU, Bethesda units; ITI, immune tolerance induction.

ancludes prothrombin complex concentrate, recombinant factor VIla and factor VIII.

${ }^{\mathrm{b}}$ Includes Native American, Alaskan Native, Native Hawaiian, other Pacific Islanders and unknown.

${ }^{c}$ No participants under $5 \mathrm{BU}$.

\subsection{Data collection and analysis}

All outcomes, except for hospitalizations, were recorded by participants using an electronic, handheld device provided during the week 1 visit and before administration of study medication. Participants were asked to record all HRQoL and health status outcomes every 4 weeks. In addition to scheduled assessments, they were asked to complete the EQ-5D-5L on any day during which bleeding occurred.

Demographic data, medical history and hospitalizations were collected from participants' medical records on an eCRF by clinicians. Analysis of covariance (model included treatment group, baseline score and the treatment-by-baseline interaction term as covariates) was used to compare the adjusted mean scores for Haem-A-QoL "Physical Health" and "Total" score and the EQ-5D-5L IUS and EQ-VAS between Arms A and B which were included as type 1 error-controlled secondary endpoints. The analysis of EQ-5D-5L IUS and EQ-VAS when a bleed occurred (unscheduled assessments) as compared with when no bleed occurred (monthly scheduled assessments) only included participants who had at least one scheduled and one unscheduled assessment.

Cumulative distribution function (CDF) plots of the change from baseline to week 25 in Haem-A-QoL "Physical Health" domain and "Total" scores were calculated for Arms A and B.
The proportion of participants achieving an improvement at week 25 larger than a previously published responder threshold was calculated for Haem-A-QoL "Total" score (-7), Haem-A-QoL "Physical Health" score (-10), EQ-5D-5L IUS (+0.07) and EQ-VAS (+7)..$^{38-41}$

The rate of participant compliance with the completion of each questionnaire (Haem-A-QoL, Haemo-QoL SF and scheduled EQ-5L-5D) at each time point was calculated by dividing the number of questionnaires by the total number expected at that time.

All analyses were based on the data cut-off used for the primary analysis. $^{29}$

\section{3 | RESULTS}

\section{1 | Study population}

The participant population for the HAVEN 1 study has been described previously ${ }^{29}$ and is summarized briefly here. Participants previously receiving episodic BPAs were randomized 2:1 to emicizumab prophylaxis (Arm A, $n=35$ ) or no prophylaxis (Arm B, $n=18$; episodic BPAs only), and 49 participants previously receiving prophylactic BPAs received emicizumab prophylaxis in Arm C. Nearly all participants had severe haemophilia at diagnosis, and $>50 \%$ in each treatment arm had experienced $\geq 9$ bleeding events in the previous 24 weeks. Participants in Arm $C$ were younger and had a higher rate of previous ITI than those in Arms A and B (Table 1). 
TAB LE 2 Haemophilia-related quality of life in adults. Mean Haem-A-QoL domain and total scores at baseline

\begin{tabular}{|c|c|c|c|}
\hline \multirow[b]{2}{*}{$\begin{array}{l}\text { Haem-A-QoL } \\
\text { domain }\end{array}$} & \multicolumn{2}{|l|}{ Previously on episodic BPAs } & \multirow{2}{*}{$\begin{array}{l}\text { Previously on prophylactic BPAs } \\
\text { Arm C: Emicizumab prophylaxis } \\
n=21 \\
\text { Mean score }{ }^{a}(95 \% \mathrm{Cl})\end{array}$} \\
\hline & $\begin{array}{l}\text { Arm A: Emicizumab prophylaxis } \\
n=29 \\
\text { Mean score }^{a}(95 \% \mathrm{Cl})\end{array}$ & $\begin{array}{l}\text { Arm B: No prophylaxis } \\
n=16 \\
\text { Mean score }^{a}(95 \% \mathrm{Cl})\end{array}$ & \\
\hline Physical Health & $52.4(44.4,60.4)$ & $57.2(46.1,68.3)$ & $59.5(48.0,71.1)$ \\
\hline Feelings & $36.9(27.2,46.5)$ & $32.4(18.0,46.8)$ & $47.9(32.6,63.2)$ \\
\hline View of Yourself & $45.3(37.6,53.1)$ & $48.8(38.7,58.8)$ & $52.1(40.9,63.4)$ \\
\hline Sports \& Leisure ${ }^{b}$ & $58.0(45.4,70.6)$ & $66.9(55.4,78.4)$ & $67.3(47.8,86.8)$ \\
\hline Work \& School ${ }^{b}$ & $36.7(23.4,49.9)$ & $42.3(31.2,53.4)$ & $50.3(33.4,67.1)$ \\
\hline $\begin{array}{l}\text { Dealing with } \\
\text { Haemophilia }\end{array}$ & $26.7(19.2,34.3)$ & $21.9(14.0,29.8)$ & $28.2(14.7,41.7)$ \\
\hline Treatment & $36.3(26.6,46.0)$ & $45.7(34.0,57.4)$ & $48.5(37.5,59.5)$ \\
\hline Future & $48.4(38.7,58.1)$ & $55.3(41.6,69.0)$ & $54.5(44.0,65.0)$ \\
\hline Family Planning $^{\mathrm{b}}$ & $27.1(9.5,44.7)$ & $44.9(21.8,68.0)$ & $43.8(9.7,77.8)$ \\
\hline $\begin{array}{l}\text { Partnership \& } \\
\text { Sexuality }\end{array}$ & $26.4(12.6,40.3)$ & $15.6(4.4,26.8)$ & $34.9(19.2,50.7)$ \\
\hline Total score & $41.1(34.0,48.3)$ & $44.6(36.7,52.5)$ & $49.4(40.4,58.4)$ \\
\hline
\end{tabular}

BPA, bypassing agents; Cl, confidence limit; Haem-A-QoL; Haemophilia Quality of Life Questionnaire for Adults.

${ }^{a}$ Scales range from 0 (no impairment) to 100 (high impairment).

bParticipants had the option to choose "not applicable" for these domains. At week 1, the number of respondents (n) for Arms A, B and C, respectively, was 20,13 and 12 for "Sports \& Leisure," 14, 11 and 8 for "Family Planning" and 20, 13 and 16 for "Work \& School".

\subsection{1 | Compliance}

Compliance with completion of all questionnaires across all time points was $\geq 90 \%$ for all outcome measures in all treatment arms.

\subsubsection{Haemophilia-specific Quality of Life in adults (Haem-A-QoL)}

Baseline mean Haem-A-QoL "Total" and domain scores are listed in Table 2.

Improvements in Haem-A-QoL domain and total scores with emicizumab prophylaxis were seen as early as week 5 , maintained through week 25 and generally similar regardless of previous treatment regimen (Figure $2 \mathrm{~A}$ and $3 \mathrm{~A}$ ). Among participants previously treated with episodic BPAs, the difference in adjusted mean scores between the emicizumab prophylaxis group (Arm A) and the no prophylaxis group (Arm B) at week 25 was statistically significant in favour of emicizumab for both "Total" ( $\Delta=14.01$; $95 \% \mathrm{Cl}$ : 5.56, 22.45; $P=0.0019)$ and "Physical Health" domain ( $\Delta=21.55 ; 95 \% \mathrm{Cl}: 7.89$, 35.22; $P=0.0029$ ) scores.

Throughout the study, a greater proportion of participants in the emicizumab prophylaxis groups (Arms $A$ and $C$ ) than the no prophylaxis group (Arm B) achieved an improvement from baseline exceeding responder thresholds ${ }^{38}$ for "Total" ( -7 points) and "Physical Health" domain (-10 points) scores (Figure S1A,B). In the cumulative distribution plots of change from baseline to week 25 for both the "Total" and "Physical Health" domain scores, there was a clear separation between Arms A and B throughout all potential levels of change (Figure $2 \mathrm{~B}$ and $3 \mathrm{~B}$ ).
Emicizumab prophylaxis also produced notable improvements from baseline in other domains of the Haem-A-QoL (Table 3), and improvements were qualitatively similar regardless of previous treatment regimen. There was no notable change in the no prophylaxis group (Arm B) in "Total" or domain scores at any time during the study.

\subsection{3 | Haemophilia-specific Quality of Life in adolescents (Haemo-QoL SF)}

The impact of emicizumab prophylaxis on Haemo-QoL SF is only presented for those participants previously treated with prophylactic BPAs (Arm C) because too few adolescents completed this questionnaire in Arms $A(n=3)$ and $B(n=2)$ for meaningful analysis.

At baseline, mean (95\% Cl) Haemo-QoL SF "Total" score was 30.7 $(24.3,37.2)$. Improvements from baseline with emicizumab prophylaxis were seen in most domains of the Haemo-QoL SF and in the "Total" score (Table 4). Improvements were seen as early as week 5 and maintained through week 25.

\subsection{4 | Health status}

At baseline, mean (95\% Cl) EQ-VAS scores were 70.7 (63.6, 77.9), $76.3(69.1,83.5)$ and $75.8(68.4,83.2)$ in Arms A, B and C, respectively. Baseline mean (95\% CI) EQ-5D-5L IUS scores were $0.72(0.63$, 0.81), 0.67 (0.52, 0.82) and $0.74(0.67,0.81)$ in Arms A, B and C, respectively. For some dimensions of the EQ-5D-5L (eg, anxiety), a considerable number of participants reported "never" having a 
(A) Mean 'Total' score over time

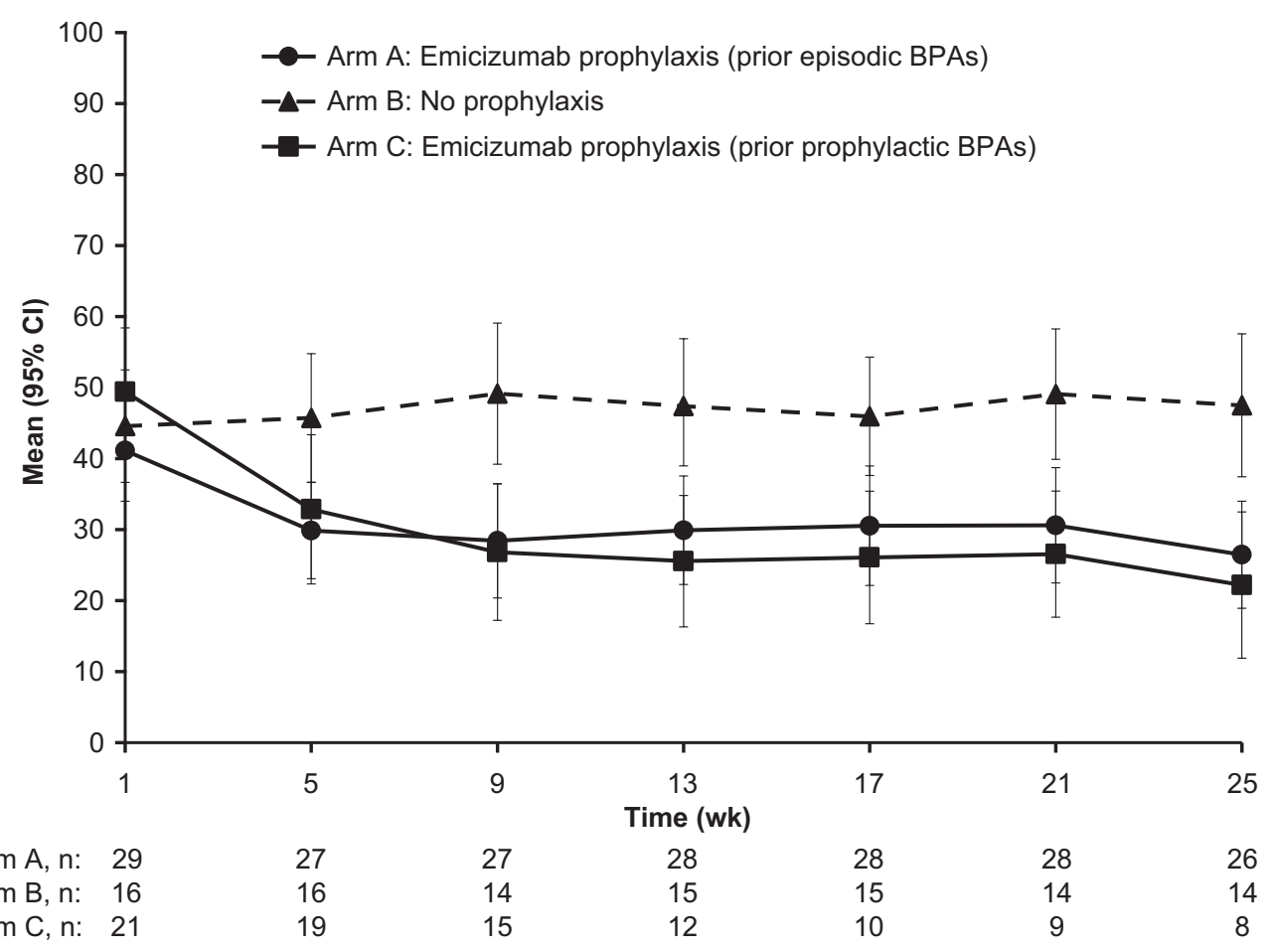

(B) Cumulative distribution plot of transformed 'Total' change scores from baseline to week 25

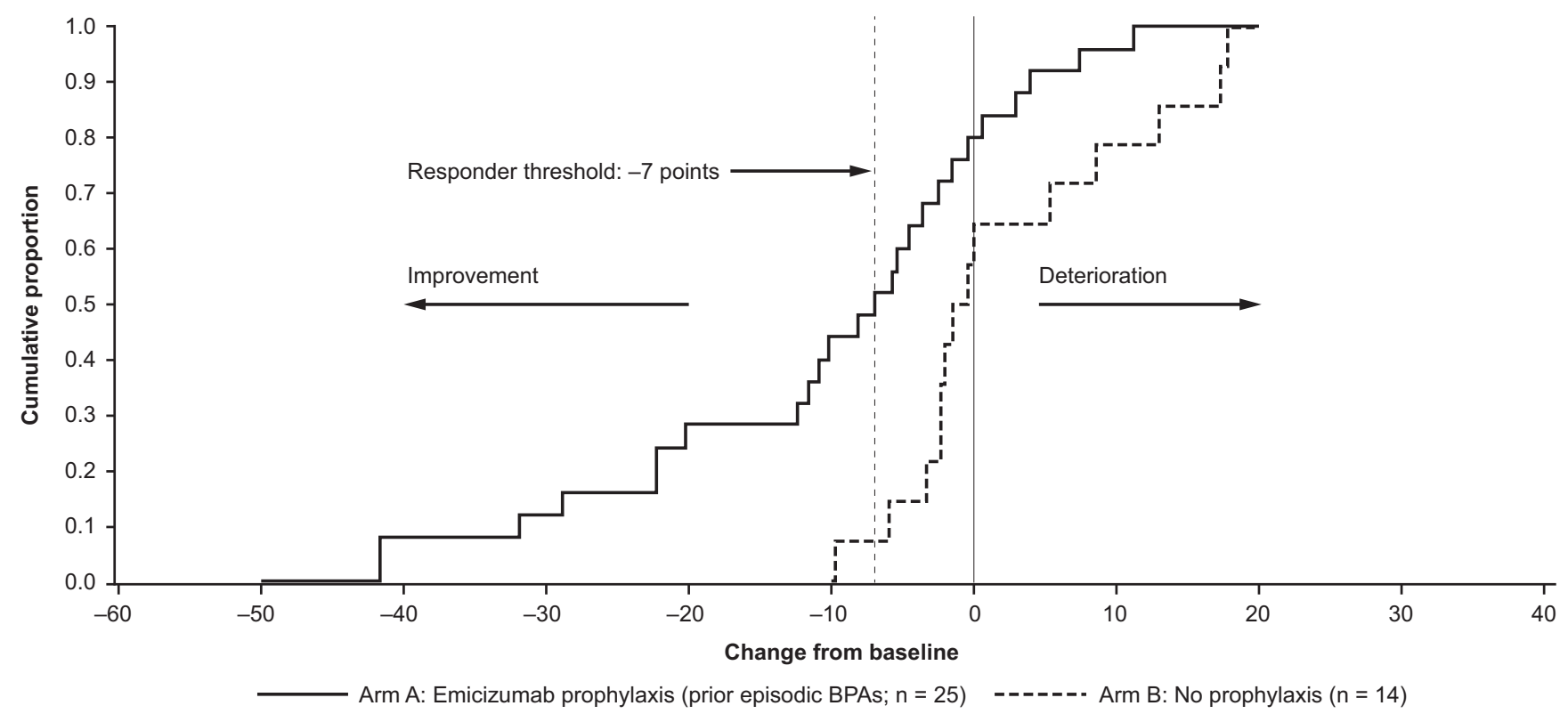

FIG URE 2 Haem-A-QoL (adults) "Total" score. Scale ranges from 0 (no impairment) to 100 (high impairment). BPA, bypassing agent; Cl, confidence interval; Haem-A-QoL, Haemophilia Quality of Life Questionnaire for Adults; QW, once a week. A. Mean score over time. B. Cumulative distribution plot of transformed "Total" change scores from baseline to week 25

problem at baseline, and this may have limited the ability to detect an improvement in some items.

Improvements in EQ-VAS and EQ-5D-5L IUS with emicizumab prophylaxis were seen as early as week 5 , maintained through week 25 , and similar regardless of previous treatment regimen (Figure 4). In the no prophylaxis group (Arm B), EQ-VAS and EQ-5D-5L IUS scores remained near baseline levels or decreased slightly during the study.
Among participants previously treated with episodic BPAs, the difference in adjusted mean scores between the emicizumab prophylaxis group (Arm A) and the no prophylaxis group (Arm B) at week 25 was statistically significant in favour of emicizumab for both the EQ$\operatorname{VAS}(\Delta=-9.72 ; 95 \% \mathrm{Cl}:-17.6,-1.82 ; P=0.0171)$ and IUS $(\Delta=-0.16$, $95 \% \mathrm{Cl}:-0.25,-0.07 ; P=0.0014)$. Throughout the study, a greater proportion of participants in the emicizumab prophylaxis groups 
(A) Mean 'Physical Health' score over time

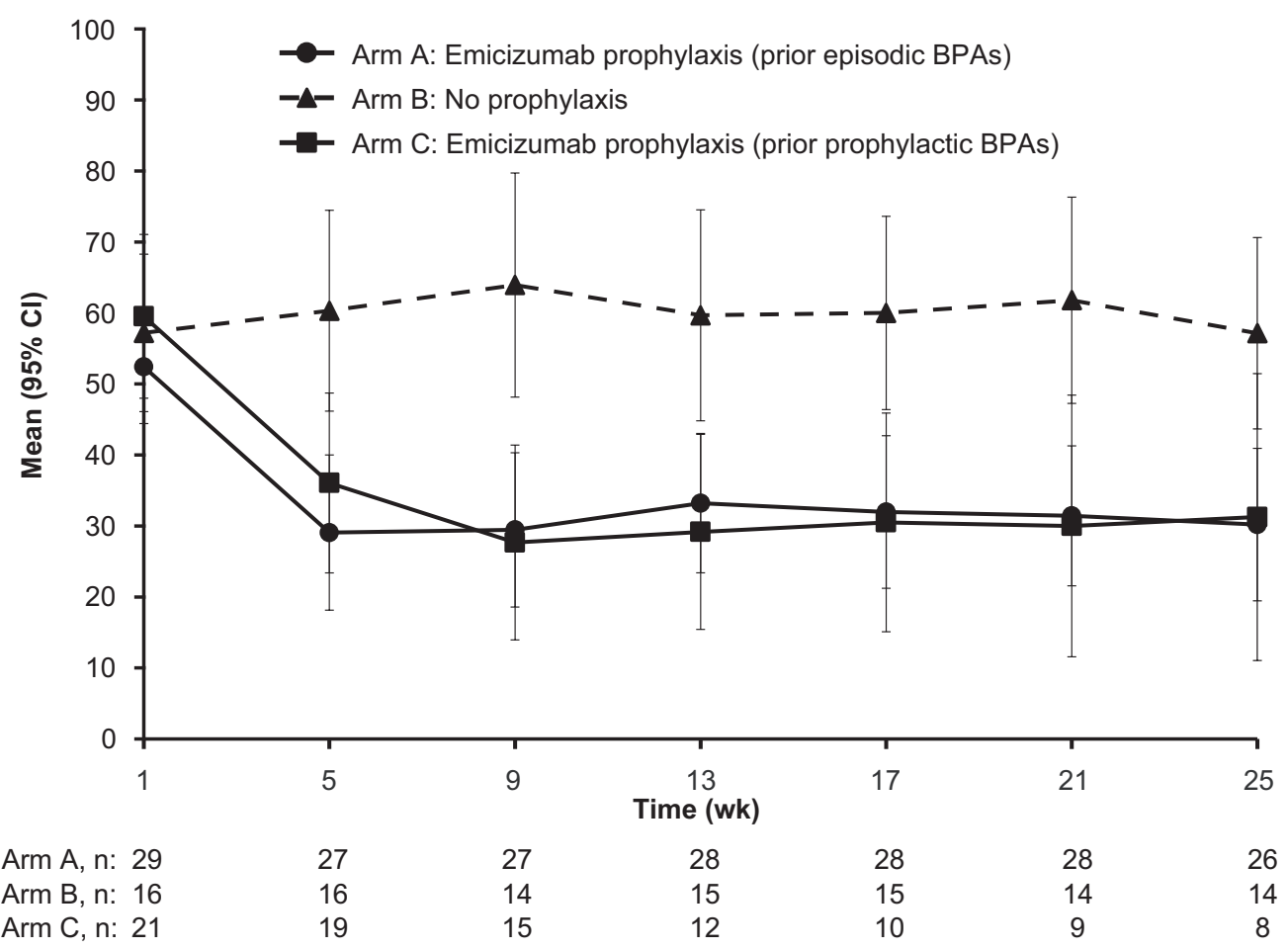

(B) Cumulative distribution plot of transformed 'Physical Health' domain change scores from baseline to week 25

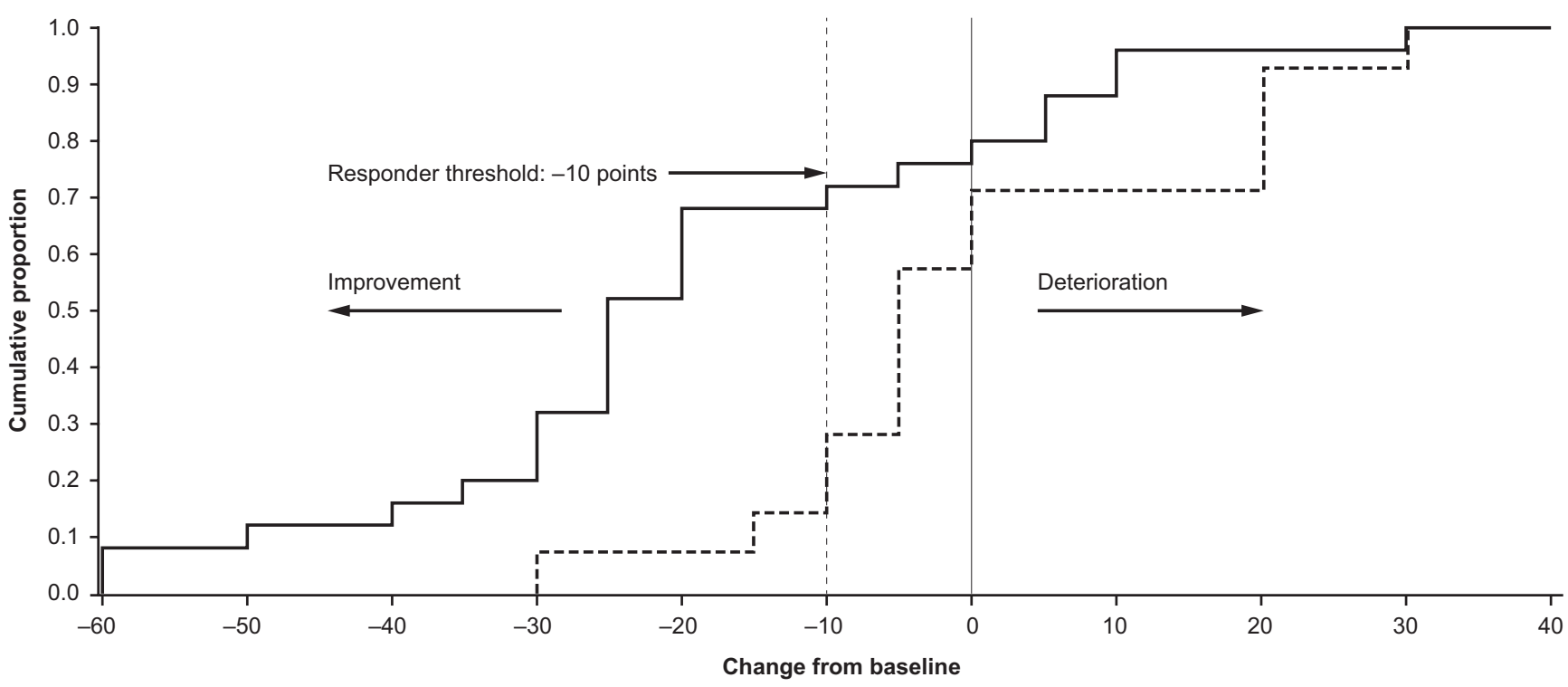

Arm A: Emicizumab prophylaxis (prior episodic BPAs; $n=25$ ) - -.--.-. Arm B: No prophylaxis $(n=14)$

FIG URE 3 Haem-A-QoL (adults) Physical Health Domain score. Scale ranges from 0 (no impairment) to 100 (high impairment). BPA, bypassing agent; $\mathrm{Cl}$, confidence interval; Haem-A-QoL, Haemophilia Quality of Life Questionnaire for Adults; QW, once a week. A, Mean score over time. B, Cumulative distribution plot of transformed "Physical Health" domain change scores from baseline to week 25

(Arms $\mathrm{A}$ and $\mathrm{C}$ ) than in the no prophylaxis group (Arm B) achieved an improvement exceeding responder thresholds (Figure S1C,D). ${ }^{39,40}$

Mean EQ-VAS and EQ-5D-5L IUS scores were generally lower (worse) on days when bleeding occurred than on regularly scheduled reporting days (Figure S2).

\subsection{5 | Work/school absences and hospitalizations}

The number of participants working before and during the study was 11 in the emicizumab prophylaxis group (Arm A) and 7 in the no prophylaxis group (Arm B). In the 4 weeks before study entry, the 
TAB LE 3 Effect of emicizumab prophylaxis on haemophilia-related quality of life in adults. Mean change from baseline to week 25 in Haem-A-QoL domain and total scores ${ }^{\text {a }}$

\begin{tabular}{|c|c|c|c|}
\hline \multirow[b]{2}{*}{ Domain } & \multicolumn{2}{|l|}{ Previously on episodic BPAs } & \multirow{2}{*}{$\begin{array}{l}\text { Previously on prophylactic BPAs } \\
\text { Arm C: Emicizumab prophylaxis } n=8^{b} \\
\text { Mean } \Delta(95 \% \mathrm{Cl})\end{array}$} \\
\hline & Mean $\Delta(95 \% \mathrm{Cl})$ & Mean $\Delta(95 \% \mathrm{Cl})$ & \\
\hline Feelings & $-14.8(-25.9,-3.6)$ & $6.7(-1.2,14.6)$ & $-14.1(-35.1,7.0)$ \\
\hline View of Yourself & $-12.2(-19.9,-4.6)$ & $2.5(-6.0,11.0)$ & $-6.3(-21.0,-8.5)$ \\
\hline Work \& School ${ }^{d}$ & $-13.1(-20.8,-5.3)$ & $8.3(-9.8,26.5)$ & $-21.9(-46.3,2.6)$ \\
\hline Dealing with Haemophilia & $-4.0(-11.7,3.7)$ & $8.3(-2.0,18.7)$ & $-20.8(-35.7,-5.9)$ \\
\hline Treatment & $-9.3(-16.5,-2.0)$ & $2.2(-4.6,9.1)$ & $-24.6(-50.1,0.9)$ \\
\hline Future & $-13.8(-23.6,-4.0)$ & $-2.5(-15.3,10.3)$ & $-17.5(-36.1,1.1)$ \\
\hline
\end{tabular}

BPA, bypassing agent; $\mathrm{Cl}$, confidence interval; Haem-A-QoL, Haemophilia-Specific Quality of Life Questionnaire for Adults; NE, not evaluable.

${ }^{a}$ Scales range from 0 (no impairment) to 100 (high impairment); negative values indicate an improvement from baseline.

${ }^{b}$ Only includes those participants with results at both baseline and week 25.

"Responder thresholds defined for "Physical Health" (-10), "Sports and Leisure" (-10) and "Total" score $(-7) .{ }^{38}$

${ }^{\mathrm{d} P a r t i c i p a n t s ~ c o u l d ~ c h o o s e ~ " n o t ~ a p p l i c a b l e " ~ f o r ~ t h e s e ~ d o m a i n s . ~ T h e ~ n u m b e r ~ o f ~ p a r t i c i p a n t s ~ w i t h ~ r e s u l t s ~ a t ~ b o t h ~ b a s e l i n e ~ a n d ~ w e e k ~} 25$ in Arms A, B and C, respectively, was 15, 11 and 3 for "Sports \& Leisure," 9, 8 and 1 for "Family Planning" and 15, 9 and 6 for "Work \& School".

eOnly 1 participant had results at both baseline and week 25 .

mean proportion of missed work days $(95 \% \mathrm{Cl})$ was $5 \%(0 \%, 12 \%)$ in Arm A and 13\% (2\%, 25\%) in Arm B. This remained essentially unchanged in both groups $(7 \%$ [0\%, 15\%] and 14\% [8\%, 19\%], respectively) during the study. For participants previously treated with prophylactic BPAs (Arm C), the mean proportion of missed work days $(95 \% \mathrm{Cl})$ decreased from $9 \%(0 \%, 23 \%)$ in 4 weeks before study entry to $3 \%(0 \%, 8 \%)$ during emicizumab prophylaxis.

Only four participants in Arms A and B (previously treated with episodic BPAs) were attending school during the study. Seventeen participants previously treated with prophylactic BPAs (Arm C) were attending school, and the mean proportion of missed school days $(95 \% \mathrm{Cl})$ was $28 \%(6 \%, 50 \%)$ during 4 weeks before study entry and $5 \%(0 \%, 11 \%)$ during emicizumab prophylaxis.

The mean $(95 \% \mathrm{Cl})$ number of days hospitalized was $1.9(0.0,5.1)$ with emicizumab prophylaxis (Arm A), $4.2(0.0,8.9)$ with no prophylaxis (Arm B) and $0.7(0.0,1.5)$ with emicizumab prophylaxis in participants previously treated with prophylactic BPAs (Arm C).

\section{DISCUSSION}

The results of this analysis demonstrate that participants receiving once weekly emicizumab prophylaxis in HAVEN 1 experienced statistically significant and clinically meaningful improvements in haemophilia-specific QoL and overall health status. On all measures, improvements were seen as early as week 5 and maintained through week 25. Moreover, improvements from baseline were similar regardless of whether previous BPA use was episodic or prophylactic. These improvements with emicizumab were likely due to a combination of substantial reductions in bleeding, the low rate of mostly mild adverse events and the once weekly subcutaneous dosing regimen. ${ }^{29}$

TAB LE 4 Effect of emicizumab prophylaxis on haemophiliarelated quality of life in adolescents. Mean change from baseline to week 25 in Haemo-QoL SF domain and "Total" scores ${ }^{a}$

\begin{tabular}{|l|l|} 
& $\begin{array}{l}\text { Previously on } \\
\text { prophylactic BPAs }\end{array}$ \\
\cline { 2 - 2 } & $\begin{array}{l}\text { Arm C: Emicizumab } \\
\text { prophylaxis } \\
\mathrm{n}=13^{\mathrm{b}}\end{array}$ \\
\hline Domain & \begin{tabular}{l} 
Mean $\Delta(95 \% \mathrm{Cl})$ \\
\hline Physical Health
\end{tabular} \\
\hline Feelings & $-24.5(-32.8,-16.3)$ \\
\hline View of Yourself & $-3.9(-13.4,5.7)$ \\
\hline Family & $-12.0(-25.1,1.1)$ \\
\hline Friends & $-15.9(-31.0,-0.7)$ \\
\hline Other People & $12.2(-3.8,28.2)$ \\
\hline Sports \& School & $-12.5(-20.2,-4.8)$ \\
\hline Dealing with Haemophilia & $-21.2(-34.0,-8.3)$ \\
\hline Treatment & $-8.2(-17.2,0.9)$ \\
\hline Total score & $-11.1(-18.5,-3.6)$ \\
\hline
\end{tabular}

BPA, bypassing agent; $\mathrm{Cl}$, confidence interval; Haem-A-QoL, Haemophilia-Specific Quality of Life Assessment for Children.

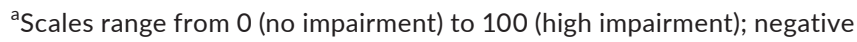
values indicate an improvement from baseline.

${ }^{b}$ Only calculated for those participants with results at both baseline and week 25. 
During emicizumab prophylaxis in HAVEN 1, mean Haem-A-QoL and Haemo-QoL SF "Total" and "Physical Health" domain scores improved from near the middle of the scale to below the lower third of the scale regardless of previous treatment regimen, suggesting a marked improvement in haemophilia-related QoL. Improvements seen in Haem-A-QoL "Work \& School" and "Sports \& Leisure" domains and the Haemo-QoL SF "Sports \& School" domain, as well as the low rate of hospitalizations and work or school absences, suggest an overall improvement in the ability to engage in activities of daily life. This is supported by the high rate of participants achieving responder thresholds on Haem-A-QoL "Total" and "Physical Health" domain scores. Currently, there is no consensus on how changes in HRQoL outcomes should be interpreted, but the responder thresholds used in this study provide an estimate of notable improvements that, when combined with other findings, suggest meaningful improvements in participants' lives. ${ }^{38}$ Further longitudinal assessments are needed, however, to quantify how the HRQoL changes observed with emicizumab prophylaxis correspond to real-life improvements.

In addition to improvements in HRQoL, emicizumab prophylaxis resulted in statistically significant improvements in overall health status as measured by the EQ-VAS and EQ-5D-5L IUS. As has been seen in other studies, ${ }^{24,42}$ EQ-5D-5L IUS scores were generally

(A) Mean EQ-VAS over time

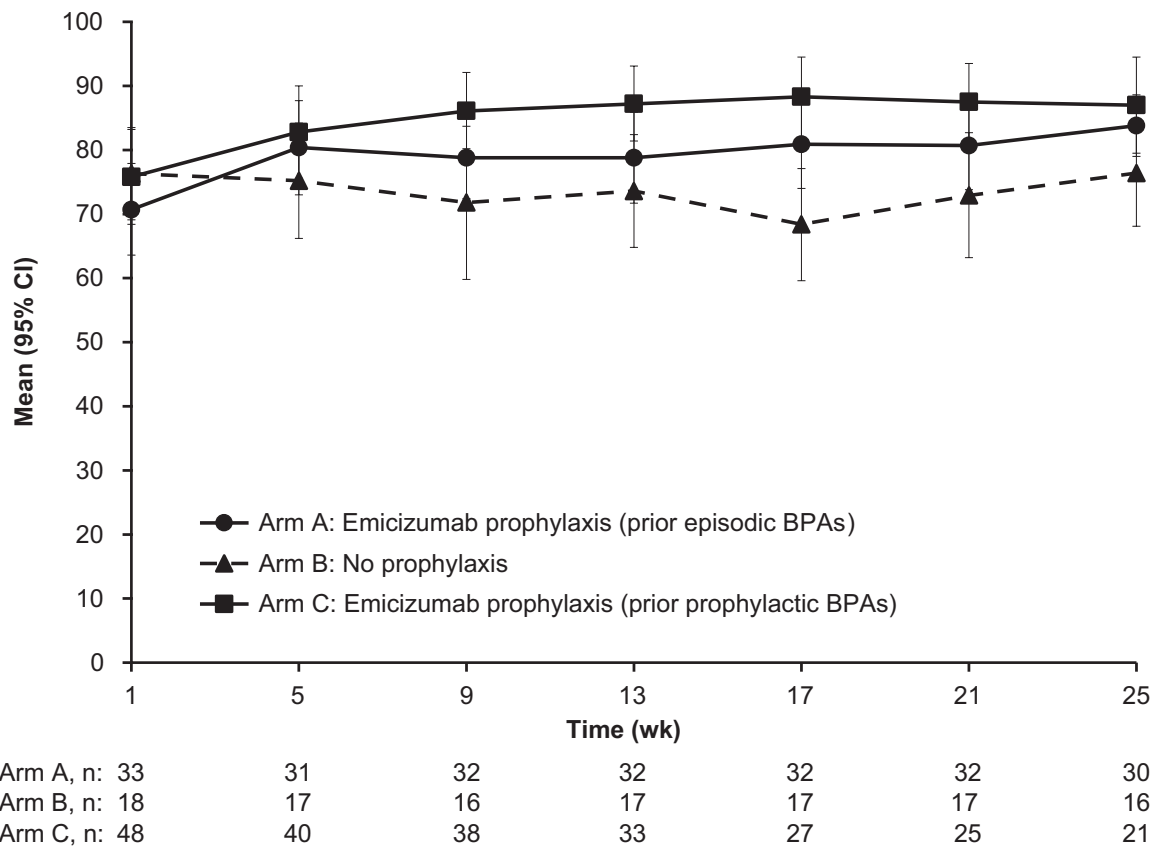

FIGURE 4 Health status as measured by the European Quality of Life 5Dimensions 5-levels (EQ-5D-5L) visual analogue scale (EQ-VAS) and index utility score (IUS). On the EQ-VAS, scores ranged from 0 (worst imaginable) to 100 (best imaginable). On the IUS, scores ranged from -0.594 (extreme problems on all dimensions) to 1 (no problems on all dimensions). BPA, bypassing agent; $\mathrm{Cl}$, confidence interval. A, Mean EQ-VAS over time. B, Mean EQ-5D-5L IUS over time
Arm C, n: 48

(B) Mean EQ-5D-5L IUS over time

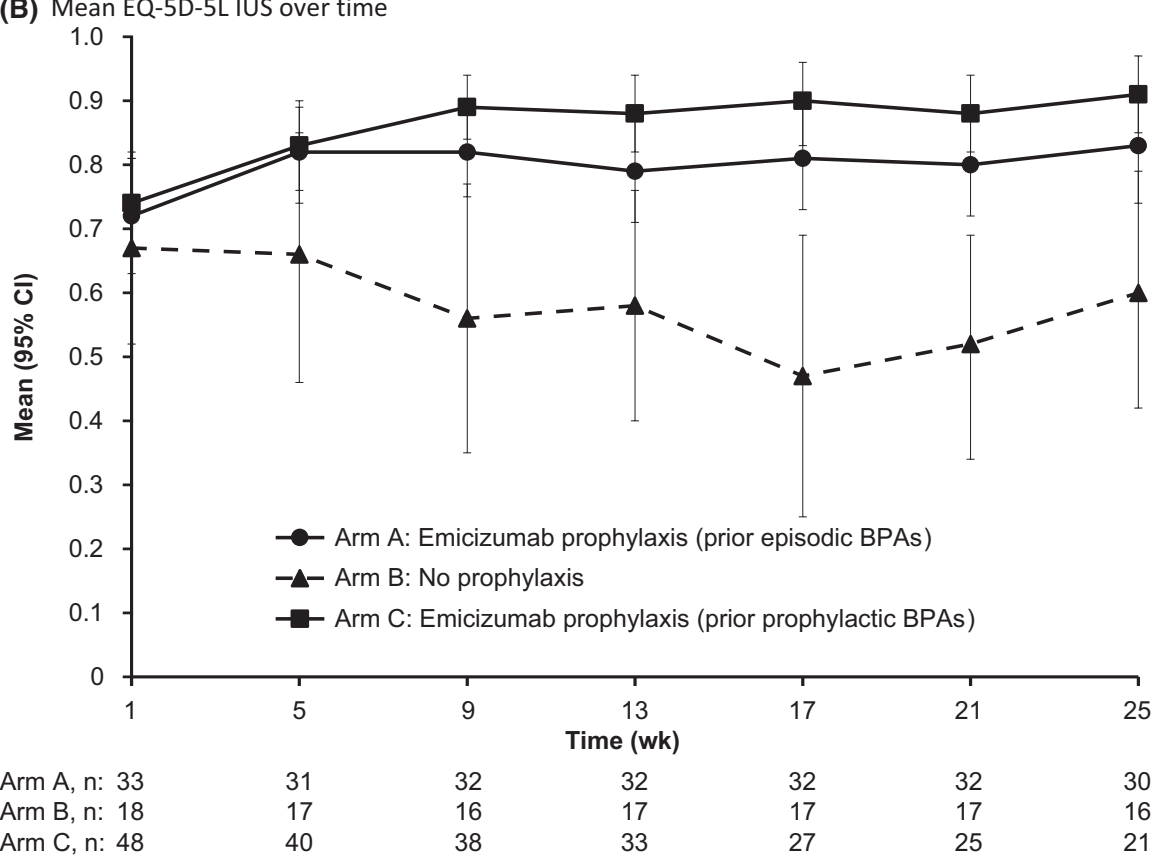


worse on the days on which bleeding occurred than on regularly scheduled reporting days.

The strengths of this study include the use of psychometrically validated, haemophilia-specific and age-appropriate HRQoL instruments. $^{33,43}$ The inclusion of overall health status measures that are not disease-specific provides a valuable complement to the disease-specific outcomes. The separate analyses for participants on different previous treatment regimens are also a strength, providing insights on how switching to emicizumab prophylaxis affected outcomes for participants with different treatment histories. This study was limited by the small number of adolescents in Arms A and B, making it difficult to evaluate the impact of emicizumab prophylaxis versus no prophylaxis on HRQoL and school attendance in this age group.

\section{5 | CONCLUSIONS}

This analysis of HAVEN 1 demonstrates that the substantial reductions in bleeding seen with emicizumab prophylaxis ${ }^{29}$ were accompanied by substantial and meaningful improvements in the daily lives of PwHA with inhibitors. Improvements in haemophiliaspecific QoL and overall health status were apparent as early as the first assessment after the start of treatment and maintained throughout the study. Importantly, improvements among participants previously treated with prophylactic BPAs mirrored the improvements seen in participants previously on episodic BPAs. These findings suggest that emicizumab prophylaxis can help meet the needs of PwHA with inhibitors for effective, less burdensome prophylaxis.

\section{ACKNOWLEDGEMENTS}

Writing assistance was provided by Amy Lindsay, $\mathrm{PhD}$, Envision Pharma Group, and funded by F. Hoffmann-La Roche Ltd.

\section{DISCLOSURES}

JO has received reimbursement for attending symposia/congresses and/or honoraria for speaking/consulting and/or funds for research from Bayer, Biogen, Biotest, Chugai, CSL Behring, Grifols, Novo Nordisk, Octapharma, Pfizer, Roche, Shire and Swedish Orphan Biovitrum. JM has received research grants from Bayer, CLS, Novo Nordisk, Pfizer and Roche; member of scientific advisory committee of CSL Behring, Chugai, Novo Nordisk, Roche, Shire and Spark; member of speaker bureau of Alnylam, Bayer, Biogen, CLS, Roche and Shire. WB is a paid consultant to Roche. PT and JX are employees of Genentech, Inc. MUC has been a paid consultant and speaker for Roche/Genentech; a paid speaker for Bayer, Shire and Novo Nordisk; and a paid consultant for Bayer, Shire, Pfizer, Grifols, Bioverativ and HEMA Biologics; and owns stock in Alnylam. GY is a consultant for Genentech/Roche. EA is an employee of Roche. FP received consulting fees from Kedrion, LFB; speaker honoraria from educational meetings from Ablynx, Alnylam, Grifols, Roche, Shire and Sobi; advisory board for Ablynx. ES is a member of advisory boards and speaker bureau for Bayer, Bioveratin, Grifols, Kedrion, Novo Nordisk, Pfizer, Roche, Shire, Sobi, and Bioverativ, CSL Behring and Octapharma. RK-J has acted as a paid consultant for CSL Behring, Genentech/ Roche, Novo Nordisk, Pfizer and Shire; and received research funding from CSL Behring, Pfizer and Genentech. CN has acted as a paid consultant for Alnylam, Baxalta/Shire, Bayer, CSL Behring, LFB, Novo Nordisk, Pfizer, Roche and Sobi, and received research funding from Baxalta, Bayer, CSL Behring, LFB, Novo Nordisk, Octapharma, Pfizer and Sobi. CK has received personal fees from Genentech during the conduct of the study; grants and personal fees from Bayer, Novo Nordisk and Octapharma; personal fees from Bioverativ; and grants from Genentech. JW has received grant/research/clinical trial support from Amgen, Aspen, Baxalta, Baxter, Bayer, Biogen Idec, CSL Behring, Novo Nordisk, Octapharma, Roche, Sanofi and Shire. MS is a board member of the FEIBA and Advate Safety Board in Japan organized by Baxalta; has received honoraria for consultancy meetings from Baxalta, Bayer, Biogen, Chugai Therapeutic Company, CSL Behring, Kaketsuken, Pfizer and Novo Nordisk; and received unrestricted grants supporting research from Baxalta, Bayer, Chugai Pharmaceutical Company, CSL Behring, Kaketsuken, Novo Nordisk and Pfizer. SvM is a consultant for Roche.

\section{AUTHORS' CONTRIBUTIONS}

PT, EA and JX contributed to study concept and design. Data analysis was conducted by EA, who vouches for the completeness and accuracy of the data and analyses. Specific direction from the authors (JO, JM, WB, PT, MC, GY, EA, FP, ES, RK-J, CN, CK, JX, JW, MS and SVM) informed the development of the first draft of the manuscript by Envision Pharma Group (funded by F. Hoffmann-La Roche Ltd). That draft was subsequently critically reviewed by all authors and revised according to their direction. All the authors had access to the data and confirm adherence to the protocol and statistical analysis plan during the conduct of the study.

\section{ORCID}

Johannes Oldenburg (iD http://orcid.org/0000-0002-1585-4100

Johnny N. Mahlangu iD http://orcid.org/0000-0001-5781-7669

Michael U. Callaghan iD http://orcid.org/0000-0001-8742-0275

Guy Young iD http://orcid.org/0000-0001-6013-1254

Flora Peyvandi (D) http://orcid.org/0000-0001-7423-9864

Rebecca Kruse-Jarres (iD http://orcid.org/0000-0001-6547-5240

Jerzy Windyga (iD http://orcid.org/0000-0001-7877-4784

Sylvia von Mackensen (iD http://orcid.org/0000-0002-5926-0478

\section{REFERENCES}

1. Kingdon HS, Lundblad RL. An adventure in biotechnology: the development of haemophilia A therapeutics - from whole-blood 
transfusion to recombinant DNA to gene therapy. Biotechnol Appl Biochem. 2002;35:141-148.

2. Khawaji M, Astermark J, Berntorp E. Lifelong prophylaxis in a large cohort of adult patients with severe haemophilia: a beneficial effect on orthopaedic outcome and quality of life. Eur J Haematol. 2012;88:329-335.

3. Peyvandi F, Garagiola I, Seregni S. Future of coagulation factor replacement therapy. J Thromb Haemost. 2013;11:84-98.

4. Ehrenforth S, Kreuz W, Scharrer I, et al. Incidence of development of factor VIII and factor IX inhibitors in haemophiliacs. Lancet. 1992;339:594-598.

5. Gouw SC, van den Berg HM, Fischer K, et al. Intensity of factor VIII treatment and inhibitor development in children with severe hemophilia A: the RODIN study. Blood. 2013;121:4046-4055.

6. White GC 2nd, Rosendaal F, Aledort LM, et al. Definitions in hemophilia Recommendation of the scientific subcommittee on factor VIII and factor IX of the scientific and standardization committee of the International Society on Thrombosis and Haemostasis. Thromb Haemost. 2001;85:560.

7. Rocino A, Franchini M, Coppola A. Treatment and prevention of bleeds in haemophilia patients with inhibitors to factor VIII/IX. J Clin Med. 2017;6:46.

8. Lindvall K, von Mackensen S, Elmståhl S, et al. Increased burden on caregivers of having a child with haemophilia complicated by inhibitors. Pediatr Blood Cancer. 2014;61:706-711.

9. Dekoven M, Wisniewski T, Petrilla A, et al. Health-related quality of life in haemophilia patients with inhibitors and their caregivers. Haemophilia. 2013;19:287-293.

10. Konkle BA, Ebbesen LS, Erhardtsen E, et al. Randomized, prospective clinical trial of recombinant factor VIla for secondary prophylaxis in hemophilia patients with inhibitors. J Thromb Haemost. 2007;5:1904-1913.

11. Berntorp E. Differential response to bypassing agents complicates treatment in patients with haemophilia and inhibitors. Haemophilia. 2009;15:3-10.

12. Leissinger C, Gringeri A, Antmen B, et al. Anti-inhibitor coagulant complex prophylaxis in hemophilia with inhibitors. N Engl J Med. 2011;365:1684-1692.

13. Srivastava A, Brewer AK, Mauser-Bunschoten EP, et al. Guidelines for the management of hemophilia. Haemophilia. 2013;19:e1-e47.

14. Collins PW, Chalmers E, Hart DP, et al. Diagnosis and treatment of factor VIII and IX inhibitors in congenital haemophilia: (4th edition). UK Haemophilia Centre Doctors Organization. Br J Haematol. 2013;160:153-170.

15. Rocino A, Coppola A, Franchini M, et al. Principles of treatment and update of recommendations for the management of haemophilia and congenital bleeding disorders in Italy. Blood Transfus. 2014;12:575-598.

16. Brackmann HH, Lenk H, Scharrer I, Auerswald G, Kreuz W. German recommendations for immune tolerance therapy in type A haemophiliacs with antibodies. Haemophilia. 1999;5:203-206.

17. Valentino LA, Kempton CL, Kruse-Jarres R, et al. US Guidelines for immune tolerance induction in patients with haemophilia a and inhibitors. Haemophilia. 2015;21:559-567.

18. Colvin BT, Astermark J, Fischer K, et al. European principles of haemophilia care. Haemophilia. 2008;14:361-374.

19. Kempton CL, Meeks SL. Toward optimal therapy for inhibitors in hemophilia. Blood. 2014;124:3365-3372.

20. Rocino A, Cortesi PA, Scalone L, et al. Immune tolerance induction in patients with haemophilia a and inhibitors: effectiveness and cost analysis in an European Cohort (The ITER Study). Haemophilia. 2016;22:96-102.

21. Ettingshausen CE, Kreuz W. Early long-term FEIBA prophylaxis in haemophilia $A$ patients with inhibitor after failing immune tolerance induction: a prospective clinical case series. Haemophilia. 2010;16:90-100.

22. Morfini M, Haya S, Tagariello G, et al. European study on orthopaedic status of haemophilia patients with inhibitors. Haemophilia. 2007;13:606-612.

23. Brown TM, Lee WC, Joshi AV, Pashos CL. Health-related quality of life and productivity impact in haemophilia patients with inhibitors. Haemophilia. 2009;15:911-917.

24. Mahlangu J, Oldenburg J, Callaghan MU, et al. Health status, health-related quality of life and work/school attendance in patients with haemophilia A with inhibitors: A prospective, multicentre, non-interventional study (NIS). Haemophilia. 2017;23 (Suppl. 2): 84.

25. Scalone L, Mantovani LG, Mannucci PM, Gringeri A. COCIS Study Investigators. Quality of life is associated to the orthopaedic status in haemophilic patients with inhibitors. Haemophilia. 2006;12:154-162.

26. Gringeri A, Mantovani LG, Scalone L, Mannucci PM, COCIS Study Group. Cost of care and quality of life for patients with hemophilia complicated by inhibitors: the COCIS Study Group. Blood. 2003;102:2358-2363.

27. Kitazawa $T$, Igawa $T$, Sampei Z, et al. A bispecific antibody to factors IXa and X restores factor VIII hemostatic activity in a hemophilia A model. Nat Med. 2012;18:1570-1574.

28. Uchida N, Sambe T, Yoneyama K, et al. A first-in-human phase 1 study of ACE910, a novel factor VIII-mimetic bispecific antibody, in healthy subjects. Blood. 2016;127:1633-1641.

29. Oldenburg J, Mahlangu JN, Kim B, et al. Emicizumab prophylaxis in hemophilia A with inhibitors. N Engl J Med. 2017;377:809-818.

30. von Mackensen S, Gringeri A, Ravera S, et al. Validation of the haemophilia-specific quality of life questionnaire for adult patients with haemophilia (Haem-A-QoL). Haematologica. 2005;90:115-116.

31. von Mackensen S, Gringeri A. Quality of life in hemophilia. In: Preedy VR, Watson RR, eds. Handbook of Disease Burdens and Quality of Life Measures. New York, NY: Springer; 2010:1895-1920.

32. von Mackensen S, Campos IG, Acquadro C, Strandberg-Larsen M. Cross-cultural adaptation and linguistic validation of age-groupspecific haemophilia patient-reported outcome (PRO) instruments for patients and parents. Haemophilia. 2013;19:e73-e83.

33. von Mackensen S, Bullinger MHaemo-QoL Group. Development and testing of an instrument to assess the Quality of Life of Children with Haemophilia in Europe (Haemo-QoL). Haemophilia. 2004;10:17-25.

34. The EuroQol Group. EuroQol-a new facility for the measurement of health-related quality of life. Health Policy. 1990;16:199-208.

35. Herdman M, Gudex C, Lloyd A, et al. Development and preliminary testing of the new five-level version of EQ-5D (EQ-5D-5L). Qual Life Res. 2011;20:1727-1736.

36. Janssen MF, Pickard AS, Golicki D, et al. Measurement properties of the EQ-5D-5L compared to the EQ-5D-3L across eight patient groups: a multi-country study. Qual Life Res. 2013;22:1717-1727.

37. Devlin NJ, Shah KK, Feng Y, Mulhern B, van Hout B. Valuing healthrelated quality of life: an EQ-5D-5L value set for England. Health Econ. 2017;27:7-22.

38. Wyrwich KW, Krishnan S, Poon JL, et al. Interpreting important health-related quality of life change using the Haem-A-QoL. Haemophilia. 2015;21:578-584.

39. Walters SJ, Brazier JE. Comparison of the minimally important difference for two health state utility measures: EQ-5D and SF-6D. Qual Life Res. 2005;14:1523-1532.

40. Pickard AS, Neary MP, Cella D. Estimation of minimally important differences in EQ-5D utility and VAS scores in cancer. Health Qual Life Outcomes. 2007;5:70. 
41. Antunes SV, Tangada S, Stasyshyn O, et al. Randomized comparison of prophylaxis and on-demand regimens with FEIBA NF in the treatment of haemophilia A and B with inhibitors. Haemophilia. 2014;20:65-72.

42. Neufeld EJ, Recht M, Sabio H, et al. Effect of acute bleeding on daily quality of life assessments in patients with congenital hemophilia with inhibitors and their families: observations from the dosing observational study in hemophilia. Value Health. 2012;15:916-925.

43. von Mackensen $S$, Eldar-Lissai $A$, Auguste $P$, et al. Measurement properties of the Haem-A-QoL in haemophilia clinical trials. Haemophilia. 2017;23:383-391.

\section{SUPPORTING INFORMATION}

Additional supporting information may be found online in the Supporting Information section at the end of the article.
How to cite this article: Oldenburg J, Mahlangu JN, Bujan W, et al. The effect of emicizumab prophylaxis on health-related outcomes in persons with haemophilia A with inhibitors: HAVEN 1 Study. Haemophilia. 2019;25:33-44. https://doi. org/10.1111/hae.13618 